\title{
Miasis cutánea por Cochliomyia hominivorax (Coquerel) (Díptera: Calliphoridae) en el Hospital Universidad del Norte, Soledad, Atlántico
}

\author{
Napoleón de la Ossa1, Luis Eduardo Castro', Lila Visbal ², Ana María Santos², Esther Díaz², \\ Claudia M.E. Romero-Vivas ${ }^{1}$ \\ 1 Grupo de Investigaciones en Enfermedades Tropicales, Departamento de Medicina, División Ciencias de \\ la Salud, Universidad del Norte, Barranquilla, Colombia \\ 2 Servicio de Pediatría, Hospital Universidad del Norte, Universidad del Norte, Barranquilla, Colombia
}

La miasis humana es el parasitismo de órganos y tejidos producido por especies de larvas del orden Díptera. El diagnóstico se realiza con base en hallazgos clínicos y se confirma con estudios entomológicos. Se presenta el caso de una niña de siete años de edad que fue llevada por su padre al servicio de urgencias por presentar fiebre asociada a una lesión abscedada en el cuero cabelludo, con salida espontánea de larvas. Como hallazgo en el examen físico se reportó pediculosis grave. La paciente fue hospitalizada y tratada con clindamicina, gentamicina e ivermectina, y mostró mejoría de sus condiciones clínicas. Se dio alta médica al cuarto día de estancia hospitalaria. Se recolectaron larvas en estadio dos de Cochliomyia hominivorax (Diptera: Calliphoridae) directamente del área lesionada, observándose la asociación miasis-pediculosis; por lo tanto, se alerta a los trabajadores del área de la salud del riesgo potencial que representa la pediculosis para el desarrollo de la miasis.

Palabras clave: miasis, infección por Cochliomyia hominivorax, ivermectina, Colombia.

Cutaneous myiasis by Cochliomyia hominivorax (Coquerel) (Díptera Calliphoridae) in Hospital Universidad del Norte, Soledad, Atlántico

Human myiasis is the parasitism of human tissues by fly larvae. Diagnoses are based on clinical pattern of tissue damage and presence of insect stages. Herein, a case myiasis is described in a seven-year-old female child. She presented with fever associated with abscessed scalp lesions containing exposed larvae. Severe pediculosis was also observed. The patient was hospitalized and treated with clindamycin, gentamicin (for bacterial secondary infections) and ivermectin (treatment for lice) after which the patient showed clinical improvement and was discharged four days later. Since human myiasis can be caused by a number of different species, larvae were collected from the patient and identified as those of Cochliomyia hominivorax (Diptera: Calliphoridae). Because other cases of coinfestation of flies and lice are on record, health workers are to be alerted about the possible pediculosis-myasis risk..

Key words: myiasis, screw worm infection, ivermectin, Colombia.

Lamiasis es el parasitismo producido porlarvas de moscas que infectan tejidos y órganos en humanos y animales. Este parasitismo ha tenido un gran impacto económico por la implementación de

\section{Correspondencia:}

Claudia M.E. Romero-Vivas, Grupo de Investigaciones en Enfermedades Tropicales, Departamento de Medicina, División Ciencias de la Salud, km 5 antigua vía a Puerto Colombia, Barranquilla, Atlántico.

Teléfono: (5) 350 9478; fax: (5) 3598852.

clromero@uninorte.edu.co

Recibido:11/06/08; aceptado:09/10/08 estrategias de control y erradicación de estas moscas en los países de Centroamérica y Norteamérica (1).

En Colombia no se conoce una prevalencia real de la situación, pero se documentan casos aislados en la literatura científica, en los cuales un diagnóstico y un manejo oportunos permiten la rápida recuperación de los pacientes (2-6).

Los agentes etiológicos suelen variar según la localización geográfica. En África tropical, 
Cordilobya anthropophaga es causa común de miasis cutánea, mientras que en las Américas, Dermatobia hominis y Cochliomyia hominivorax son los principales agentes etiológicos $(7,8)$. Sin embargo, debido al fenómeno de las migraciones y la movilización ilegal de animales, los agentes etiológicos no se encuentran estrictamente delimitados a una región geográfica; de esta manera, se encuentra miasis por $C$. anthropophaga en las Américas $(9,10)$.

Clínicamente, las miasis humanas se dividen en cavitarias, cutáneas e intestinales. La miasis cavitaria se desarrolla en cualquier cavidad del cuerpo y toma su nombre según la localización (orbital, de boca, de nariz, vaginal o ureteral, entre otras). La miasis cutánea, a su vez, se divide en miasis de heridas y forunculosas. $C$. hominivorax, D. hominis o C. anthropophaga, entre otros, pueden ser agentes causales de ambas.

El diagnóstico de miasis cutánea es fácil de hacer ante la identificación de las larvas. La ivermectina ha resultado ser un fármaco de gran utilidad en los casos de miasis, sobre todo en el tratamiento prequirúrgico cuando se requiere una intervención. El objetivo de la presentación de este caso es alertar a la comunidad médica sobre el riesgo que tiene para los humanos el desarrollo de la miasis debido a la presencia de otros ectoparásitos, como Pediculus humanus capitis (Phthiraptera: Pediculidae).

\section{Presentación del caso}

Se trata de una paciente femenina de siete años de edad que fue llevada por su acudiente (padre) al servicio de urgencias por presentar un cuadro clínico de dos semanas de evolución, aproximadamente, caracterizado por la aparición de una masa dolorosa en la región occipital, cefalea y fiebre no cuantificada de dos días de evolución, asociados a la salida espontánea de "gusanos", según comentó el padre de la menor, motivo por el cual consultó. El padre refirió que la niña fue tratada en casa durante dos días con amoxicilina y presentó mejoría del cuadro febril.

Como antecedentes de importancia, la paciente presentó asma leve intermitente y fue sometida a una herniorrafia umbilical dos años antes.
Comentó el padre que la menor tenía el esquema de vacunación completo, sin mostrar el carné. Como antecedentes psicosociales, la paciente cursaba primero de primaria, cohabitaba con cinco personas y dormía en una hamaca con sus tres hermanos. El padre y la hermana dormían en la misma habitación. La casa poseía todos los servicios básicos (agua, alcantarillado, luz y gas), techo de eternit y piso de cemento. Comentó el padre que la madre de sus hijos murió un año antes y él estaba al cuidado de ellos.

En el examen físico se encontró la paciente conciente, orientada, hidratada y afebril, con frecuencia cardiaca de 120 latidos por minuto, frecuencia respiratoria de 18 por minuto $y$ temperatura de $37,5^{\circ} \mathrm{C}$. Tenía un peso de $21 \mathrm{~kg}$ y una talla de 1,21 m.

En la inspección se encontró normocéfala, con una tumefacción dolorosa de $15 \mathrm{~cm}$ de diámetro en la región parieto-occipital y múltiples orificios por donde salían abundantes larvas y secreción purulenta. Se encontró laceración por prurito debido a pediculosis. El resto del examen físico estaba dentro de los parámetros normales.

Se hicieron impresiones diagnósticas de: 1) miasis cutánea forunculosa; 2) pediculosis capitis; 3) sospecha de maltrato infantil teniendo como indicador la higiene personal deficiente (abandono) (11), y 4) escolar eutrófica.

Se decidió hospitalizarla e iniciar antibioticoterapia con clindamicina (30 mg/kg por día), gentamicina (6 mg/kg por día) e ivermectina oral $(200 \mu \mathrm{g} / \mathrm{kg}$ por dosis), y tratamiento concomitante con analgésicos (acetaminofén, $10 \mathrm{mg} / \mathrm{kg}$ cada 6 horas).

Se rasuró el área de la lesión y se cubrió con apósitos (figura 1). Se ordenaron curaciones diarias, radiografía de cráneo simple y valoración por cirugía plástica y por el Instituto Colombiano de Bienestar Familiar.

Los exámenes paraclínicos reportaron; leucocitos, $16.600 / \mathrm{mm}^{3}$; linfocitos, $2.200 / \mathrm{mm}^{3}$; neutrófilos, $13.800 / \mathrm{mm}^{3}$; hemoglobina, $9,8 \mathrm{mg} / \mathrm{dl}$ y hematocrito, $30 \%$; plaquetas, $677.000 / \mathrm{mm}^{3}$, y velocidad de sedimentación globular de $26 \mathrm{~mm} /$ hora.

Las radiografías de cráneo simple no evidenciaron compromiso óseo. El servicio de cirugía plástica 
decidió continuar igual manejo terapéutico y programar para un lavado quirúrgico, más desbridamiento. Durante las primeras curaciones, previo desbridamiento quirúrgico, se realizó extracción de incontables larvas muertas. La paciente manifestó dolor durante el procedimiento (figura 2).

Continuó con antibioticoterapia y se trasladó a la sala de cirugía para desbridamiento y lavado quirúrgico. La paciente toleró el procedimiento sin complicaciones (figura 3).

La niña fue trasladada a las salas de hospitalización, donde presentó evolución favorable, con disminución del edema, el eritema y el calor en la lesión y el área circunscrita. En su estancia posquirúrgica no presentó larvas sólo material serohemático en la herida. Se decidió dar alta médica al cuarto día de estancia hospitalaria, con dicloxacilina (60 mg/kg diarios) e ivermectina oral $(200 \mu \mathrm{g} / \mathrm{kg})$ en dosis única.

Ante la sospecha de maltrato infantil por abandono y negligencia, se solicitó seguimiento por psicología en su Unidad de Atención Básica y remisión al Instituto Colombiano de Bienestar Familiar.

Durante su estancia hospitalaria se recolectaron larvas directamente del área lesionada y se preservaronenalcoholal $70 \%$.Serealizóelestudio entomológico con base en claves taxonómicas (9). Por las características encontradas, se

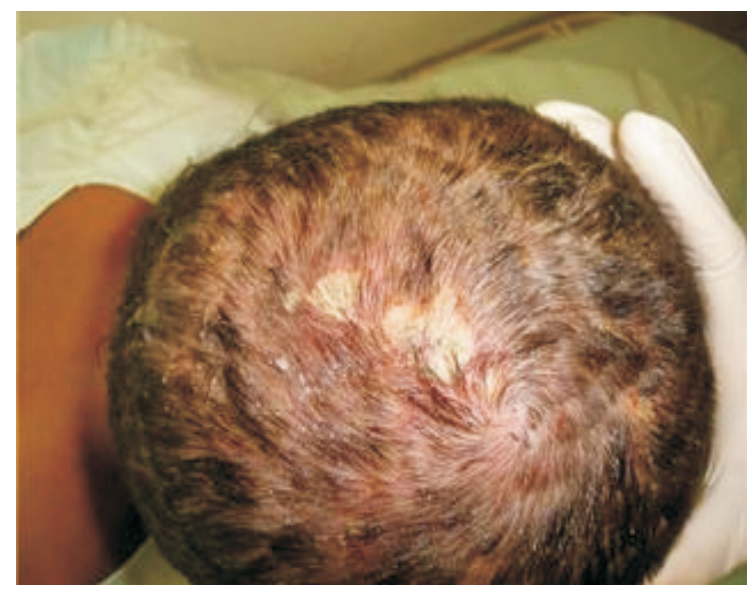

Figura 1. Lesión ulcerada cubierta por apósitos. concluyó que la larvas pertenecían a la especie C. hominivorax en estadio dos, con seis días de eclosión, aproximadamente (figura 4).

\section{Discusión}

Los primeros casos de miasis se conocen por historias anecdóticas desde los tiempos de la colonización. El primer caso de miasis cutánea fue reportado por Hope en 1840 (12). En 1935, en Texas se reportó una epidemia por $C$. hominivorax que afectó a más de un millón de animales vivos (13).

En la literatura científica se reportan casos de miasis cutánea en humanos en los países tropicales y subtropicales, desde Estados Unidos hasta Argentina; en Curazao se reportaron 25 casos en una epidemia en 1975 por $C$. hominivorax (13). En Venezuela, Argentina y Panamá (14-16) se reportan casos de miasis cutánea en humanos por $C$. hominivorax. En este último país se han hecho grandes esfuerzos para la erradicación de este díptero, con resultados satisfactorios y disminución del índice de miasis cutánea por $C$. hominivorax. (1)

En Colombia se han reportado casos aislados de miasis cutánea por $C$. homimivorax, pero no se conoce de un estudio que aporte datos de prevalencia o en el que se evalúen los factores de riesgo y el tipo de población más vulnerable (17).

En general, se conoce que las condiciones sanitarias del paciente juegan un papel importante en la predisposición a esta parasitosis. Aquellas personas con déficit en el autocuidado y el aseo personal, y con heridas abiertas, se encuentran en mayor riesgo de presentar miasis cutánea por $C$. hominivorax. Los adultos de esta especie tienen la particularidad de ser atraídos por heridas expuestas y depositar sus huevos en ellas. Las larvas de este díptero necesitan tejidos vivos para el desarrollo de su ciclo de vida (9).

Es evidente que el descuido y abandono del tutor hacia la menor fueron los factores que determinaron el riesgo de la paciente, reflejado en la presencia de otras parasitosis como la pediculosis, y es posible que la paciente desarrollara laceraciones en el cuero cabelludo 


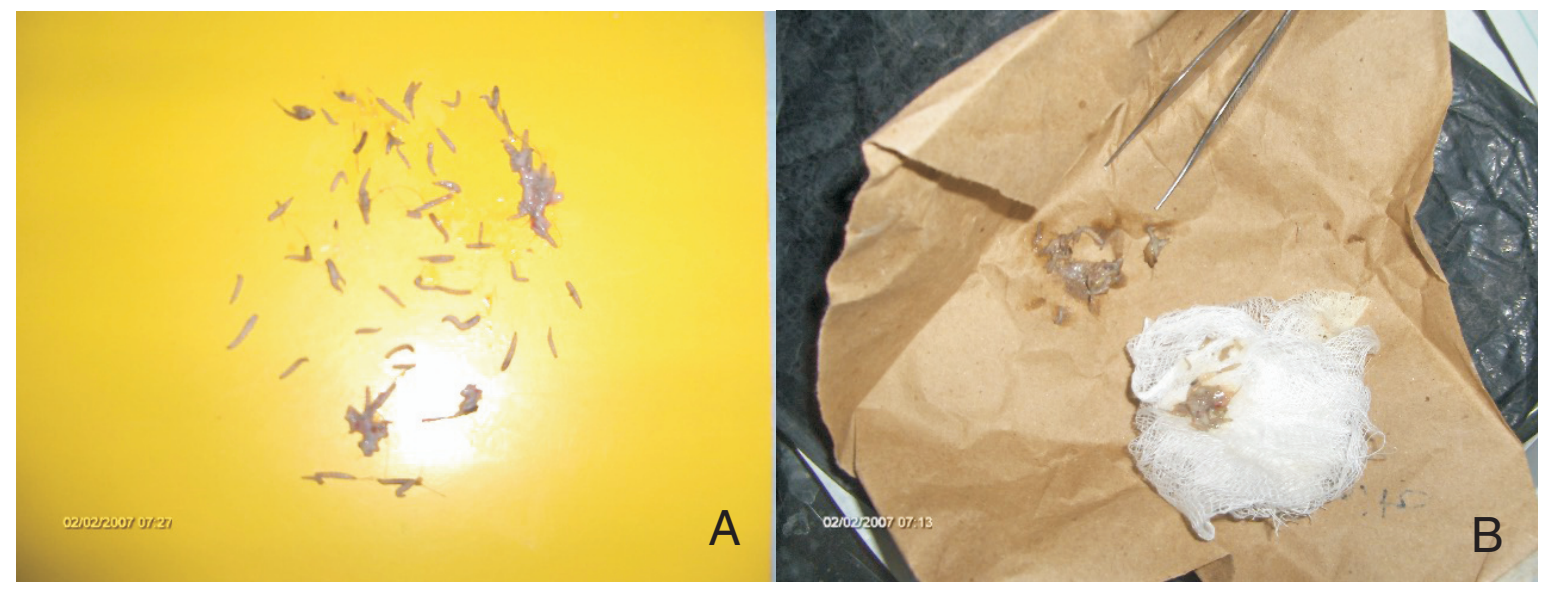

Figura 2. Extracción manual de 52 larvas de $C$. hominivorax (A) durante el proceso de curación (B).

debido al prurito intenso producido por esta parasitosis. La asociación miasis-pediculosis se ha reportado previamente en Suramérica $(18,19)$. Estas heridas son foco de infecciones secundarias, ya sean bacterianas u oportunistas parasitarias, como lo demostraron Márquez et al. en cinco áreas urbanas de Rio de Janeiro (Brasil), en donde $22,5 \%$ de los pacientes con miasis cutánea presentaba como enfermedad de base pediculosis capitis (19). Otro factor de riesgo tiene que ver con el sexo, ya que se ha evidenciado que el sexo femenino tiene predisposición para desarrollar pediculosis (20), como sucedió en nuestro caso.

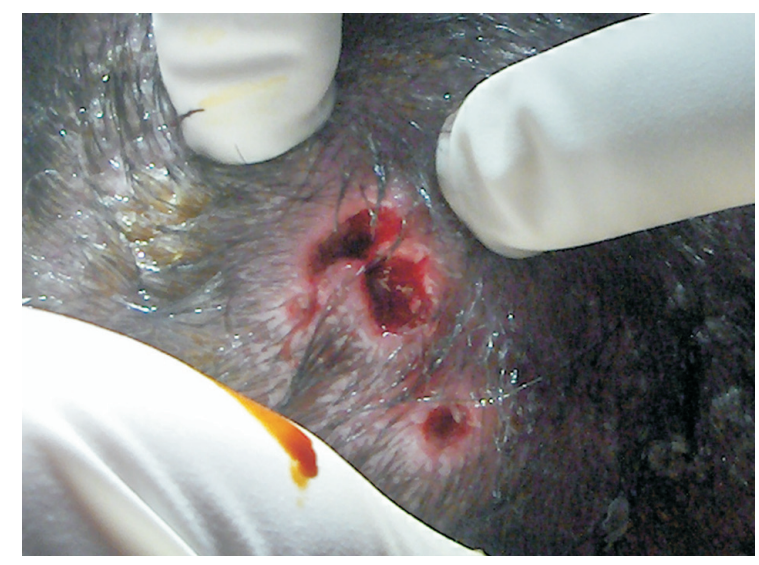

Figura 3. Herida limpia posterior desbridamiento quirúrgico. Nótese el grado de ulceración.
La leucocitosis a expensas de neutrófilos y la trombocitosis en la paciente evidenciaron una probable infección bacteriana. Se debe tomar como prioridad la extracción manual de las larvas (de ser posible) y la prevención de infecciones bacterianas secundarias (19). Se debe implementar una adecuada terapia profiláctica; la administración de clindamicina y gentamicina favorecieron la remisión del proceso infeccioso evidenciado por los parámetros del hemograma de control.

La ivermectina oral ha resultado de gran utilidad en miasis cutáneas y pediculosis capitis (21). Las curaciones posteriores evidenciaron la salida de larvas muertas y ausencia de pediculosis capitis. En nuestra paciente reportamos excelentes resultados posoperatorios tras una dosis previa de ivermectina oral $(200 \mu \mathrm{g} / \mathrm{kg})$. En otros tipos de miasis, como la orbital, se ha demostrado que el uso de ivermectina disminuye el proceso inflamatorio previo al desbridamiento, lo cual evita la enucleación en algunos casos (17).

\section{Agradecimientos}

Los autores agradecen las sugerencias al trabajo realizadas por Andrew Falconar, del Grupo de Investigaciones en Enfermedades Tropicales de la Universidad del Norte.

\section{Conflicto de intereses}

No existe conflicto de intereses. 

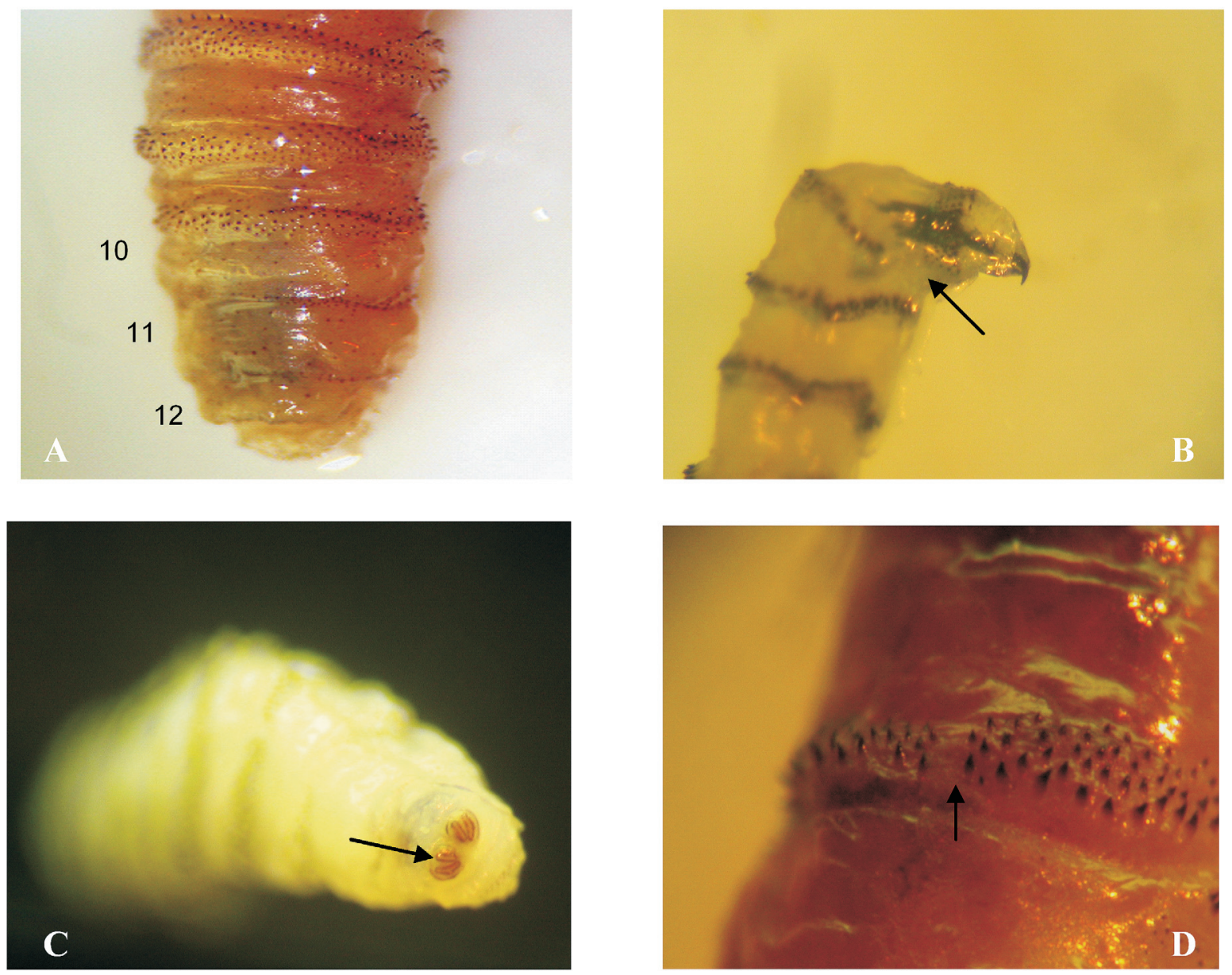

Figura 4. Características morfológicas en las larvas que permiten realizar el diagnóstico entomológico de C. hominivorax: A. Troncos traqueales dorsales con pigmentación oscura desde el segmento 12 hasta el 10. B. Morfología de esqueleto cefalofaríngeo. C. Espiráculos posteriores con anillo peritremático abierto. D. Interrupción estrecha en el dorso de la banda del segmento 10.

\section{Financiación}

Los autores no recibieron financiación para el desarrollo de este trabajo. La paciente estaba hospitalizada en el Hospital Universidad del Norte.

\section{Referencias}

1. Bermúdez SE, Espinosa JD, Cielo AB, Clavel F, Subia $\mathrm{J}$, Medianero E. Incidence of myiasis in Panamá during the eradication of Cochliomyia hominivorax (Coquerel 1858, Diptera: Calliphoridae) (2002-2005). Mem Inst Oswaldo Cruz. 2007;102:675-9.

2. Villamizar JR, Sandoval GP. Miasis ótica. Acta de Otorrinolaringología y Cirugía de Cabeza y Cuello. 2000;28:203-6.
3. Mariwalla K, Langhan M, Welch KA, Kaplan DH. Cutaneous myiasis associated with scalp psoriasis. J Am Acad Dermatol. 2007;57:s51-2.

4. Levi F, Valderrama R, Gonzalo JA. Tratamiento de miasis oral con ivermectina: notificación de tres casos causados por Cochliomyia homninivorax (Coquerel). Rev Fac Odontol Univ Antioquia. 1998;10:41-7.

5. Alarcón MA. Miasis uterina. Rev Colomb Obstet Ginecol. 1988;39:130-1.

6. Duque CS, Marrugo G, Valderrama R. Otolaryngic manifestations of myiasis. Ear Nose Throat J. 1990;69:619-22.

7. Vega-Lopez F, Chopra S. Manson's tropical diseases. 21st ed. London: Saunders; 2003. p. 374-5. 
8. Phillips PL, Welch JB, Kramer M. Seasonal and spatial distributions of adults screwworms (Diptera: Calliphoridae) in the Panama Canal Area, Republic of Panama. J Med Entomol. 2004;41:121-9.

9. Vargas M, Hall MJ. Manual para la mosca del gusano barrenador del ganado, Cochliomyia hominivorax (Coquerel). Volumen 1. Roma: Organización de las Naciones Unidas para la Agricultura y la Alimentación; 1993. p. 5-6.

10. Hendrix CM, King-Jackson DA, Wilson PM, Blagburn BL, Kindsay DS. Furunculoid myiasis in a dog caused by Cordylobia anthropophaga. J Am Vet Med Assoc. 1995;207:1187-9.

11. Loredo A, Trejo J, Castilla L. Children injured: abuse or accident? Diagnosis through indicators. Bol Med Hosp Infant Mex. 2003;60:368-79.

12. Hope FW. On insects and their larvae occasionally found in the human body. Trans R Soc Entomol. 1840;2:25671.

13. Organización de las Naciones Unidas para la Agricultura y la Alimentación. Miasis cutáneas, 2006. [Fecha de consulta: 29 de febrero de 2008]. Disponible en: http://www.rlc.fao.org/es/prioridades/transfron/miasis/ cutaneas/default.htm

14. Moissant de Román E, García ME, Quijada J, Simoes D, Marcial T. Miasis cutánea humana. Un caso clínico. Kasmera. 2004;32:12-5.
15. Duro EA, Marilvis JC, Mulieri PR. Umbilical myiasis in a human newborn. J Perinatol. 2007;27:250-1.

16. Powers NR, Yorgensen ML, Rumm PD, Souffront W. Myasis in humans: an overview and report of two cases in the Republic of Panama. Mil Med. 1996;161:495-7.

17. Osorio J, Moncada L, Molano A, Valderrama S, Gualtero S, Franco-Paredes C. Role of the ivermectin in the treatment of severe orbital myiasis due to Cochliomyia hominivorax. Clin Infec Dis. 2006;3:57-9.

18. Visciarelli EC, García SH, Salomón C, Cofre C, Costamanga S. Un caso de miasis humana por Cochliomyia hominivorax (Díptera: Calliphoridae) asociado a pediculosis en Mendoza, Argentina. Parasitol Latinoam. 2003;58:166-8.

19. Marquez AT, Mattos Mda S, Nascimento SB. Miases associadas com alguns factores sócio-econômicos em cinco áreas urbanas do Estado do Rio de Janeiro. Rev Soc Bras Med Trop. 2007;40:175-80.

20. Linardi PM, De Maria M, Botelho JR, Cunha HC, Ferreira J. Pediculose capitis: prevalência em escolares da rede municipal pública de Belo Horizonte, Minas Gerais, Brasil. Mem Inst Oswaldo Cruz. 1989;84:327-31.

21. Dourmishev AL, Dourmishev LA, Schwartz R. Ivermectin: Pharmacology and application in dermatology. Int J Dermatol. 2005;44:981-8. 